\section{Interval Valued Intuitionistic Fuzzy Evaluations for Analysis of a Student's Knowledge in University e-Learning Courses}

\author{
Taekyun Kim $^{1}$, Evdokia Sotirova ${ }^{2}$, Anthony Shannon ${ }^{3}$, Vassia Atanassova ${ }^{4}$, \\ Krassimir Atanassov ${ }^{2,4}$, and Lee-Chae Jang ${ }^{5}$ \\ ${ }^{1}$ Department of Mathematics, Kwangwoon University, Seoul, Korea \\ ${ }^{2}$ Intelligent Systems Laboratory, University "Prof. Dr. Assen Zlatarov", Burgas, Bulgaria \\ ${ }^{3}$ Warrane College, The University of New South Wales, Kensington, Australia \\ ${ }^{4}$ Department of Bioinformatics and Mathematical Modelling, Institute of Biophysics and Biomedical \\ Engineering, Bulgarian Academy of Sciences, Sofia, Bulgaria \\ ${ }^{5}$ Graduate School of Education, Konkuk University, Seoul, Korea
}

\begin{abstract}
In the paper a method is proposed for evaluation of the students' knowledge obtained in the university e-learning courses and an evaluation of the whole student class. For the assessment of the student's solution of the respective assessment units the theory of intuitionistic fuzzy sets is used, while for the class evaluation, interval valued intuitionistic fuzzy sets is used. The obtained intuitionistic fuzzy estimations reflect the degree of each student's good or poor performances, for each assessment unit. The interval valued intuitionistic fuzzy evaluations are based on the separate student's evaluations. We also consider a degree of uncertainty that represents such cases wherein the student is currently unable to solve the problem. The method presented here provides the possibility for the algorithmization of the process of forming the student's evaluations.
\end{abstract}

Keywords: e-learning, Interval valued intuitionistic fuzzy evaluations, Intuitionistic fuzzy evaluation, Model

\section{Introduction}

Within the context of e-learning, the information exchange between the education and training

Received: Jul. 10, 2018

Revised : Sep. 22, 2018

Accepted: Sep. 22, 2018

Correspondence to: Lee-Chae Jang

(Lcjang@konkuk.ac.kr)

(CThe Korean Institute of Intelligent Systems

CCThis is an Open Access article distributed under the terms of the Creative Commons Attribution Non-Commercial License (http://creativecommons.org/licenses/ by-nc/3.0// which permits unrestricted noncommercial use, distribution, and reproduction in any medium, provided the original work is properly cited. system and the student is performed electronically. The student obtains information on a given topic at his/her local electronic device. After this the student's acquisition of knowledge can be rated by asking appropriate questions and problems for solving, in order to pass on to the next topic of training.

During the process of e-learning the students have access to different training materials that can be classified as [1]:

- information units to acquire knowledge and skills;

- assessment units - task, problems, test;

- information resources - library, internet, and so on.

The purpose of the present paper is to offer assessments of the process of e-learning within 
university courses. The research is a continuation of previous investigations of the authors into the modelling of basic processes and functions of a typical university. In a series of research papers, the authors have studied some of the most important processes of functioning of universities [2-10]. In particular, Generalized Nets [11], are used to describe the process of student assessment [2, 6, 7], where the assessments can be represented in an intuitionistic fuzzy and in an interval valued intuitionistic fuzzy form. The concept of intuitionistic fuzzy set is described in details in [12,13] and of interval valued intuitionistic fuzzy set in [12].

The process of evaluation of the problems solved by students is described in [2]. Shannon et al. [5] describe the process of evaluation by lecturers of the tasks presented by students. In [6], the authors constructed a model, which describes the process of evaluation by lecturers. Next, a model that describes the standardization of the process of evaluation by lecturers was constructed [7]. In the next steps of the educational processes there are models of the evaluation of the lecturers themselves [8], and the assessment of the course [9]. In [10], the intuitionistic fuzzy assessments are used for modelling the process of e-learning of mathematics topics within university courses.

The aim of the present paper is to use the techniques of intuitionistic fuzzy sets for modelling the process of e-learning within the university educational environment.

\section{Proposed Assessment Model}

The students' knowledge assessment is implemented on two stages. First, we determine the evaluations of the assessment units for each student. Then we evaluate the final mark for each student using weight coefficients of the different assessment units and the obtained evaluations for them.

Let us consider a group of $m$ students, the students are labelled as follows: $i=1,2, \ldots, m$, and then the students have to be evaluated via $n$ assessment units, $j=1,2, \ldots, n$. The assessments, which estimate a summative account of the students' knowledge for the different problems, are formed on the basis of a set of intuitionistic fuzzy estimations $\langle\mu, \nu\rangle$ of real numbers from the set $[0,1] \times[0,1]$, related to the respective assessment units. These intuitionistic fuzzy estimations reflect the degree of each student's good performances $\mu$, or poor performances $\nu$, for each assessment unit and for them is valid that

$$
\mu+\nu \leq 1
$$

The degree of uncertainty $\pi=1-\mu-\nu$ represents such cases wherein the student is currently unable to solve the problem and needs additional information. Within the paper the ordered pairs were defined in the sense of intuitionistic fuzzy sets.

\subsection{Determination of the Students' Assessments of the Dif- ferent Units}

The way of evaluation of the different units can vary, but for some groups of themes (e.g., mathematics, informatics, physics, chemistry, etc.), the evaluations of the students' solutions of the different problems can be obtained, in general, by two cases:

Case 1: The assessment unit $j$ contains $u^{j}$ in number subtasks (questions).

In this case, the assessment unit can be in the form of a test with questions with attached possible answers "yes" and "no", or with questions with an attached list of optional answers.

Thus, the evaluation of the $i$-th student for the $j$-th assessment unit is obtained in two ways according to the following formula (1), for $i=1,2, \ldots, m, j=1,2, \ldots, n$.

$$
\left\langle\mu^{j}(i), \nu^{j}(i)\right\rangle=\left\langle\frac{r^{j}(i)}{u^{j}}, \frac{s^{j}(i)}{u^{j}}\right\rangle,
$$

where

- $r^{j}(i)$ is the number of right answers of the subtasks/questions in the assessment unit $j$,

- $s^{j}(i)$ is the number of wrong answers of the subtasks/questions in the assessment unit $j$,

- $u^{j}$ is the total number of subtasks/questions in the assessment unit $j$.

Therefore, the degree of uncertainty in this case is determined by the number of the questions which the student had not worked on.

Case 2: The assessment unit $j$, for example one task, is evaluated independently for $w^{j}$ levels.

Initially, when there has not been information obtained for the assessment unit, then the estimation is given by the initial values $\langle 0,0\rangle$. For $k \geq 0$, the current $(k)$-st estimation of the $i$-th student for the $j$-th assessment unit is obtained on the basis of the previous estimations according to the recurrence relation involved in the following formula (2), $i=1,2, \ldots, m, j=1,2$, ..., $n$.

$\left\langle\mu_{k}^{j}(i), \nu_{k}^{j}(i)\right\rangle$ 


$$
=\left\langle\frac{(k-1) \mu_{k-1}^{j}(i)+a_{p_{l}}^{j}(i)}{k}, \frac{(k-1) \nu_{k-1}^{j}(i)+b_{p_{l}}^{j}(i)}{k}\right\rangle,
$$

where

- $\left\langle\mu_{k-1}^{j}(i), \nu_{k-1}^{j}(i)\right\rangle$ is the previous estimation of the $j$-th assessment unit of the $i$-th student on the basis of the solutions of the already solved subtasks in the completed levels,

- $\left\langle a_{p_{l}}^{j}(i), b_{p_{l}}^{j}(i)\right\rangle$ is the estimation of the level $p_{l}$ of the $j$ th assessment unit of the $i$-th student, for $a_{p_{l}}^{j}(i), b_{p_{l}}^{j}(i) \in$ $[0,1], a_{p_{l}}^{j}(i)+b_{p_{l}}^{j}(i) \leq 1$, and $l=1,2, \ldots, w$.

- $a_{p_{l}}^{j}(i)$ and $b_{p_{l}}^{j}(i)$ are calculated according to (3) and (4) in the following way:

$$
\begin{aligned}
& \int \frac{c_{l}^{j}(i)+d_{l}^{j}(i)}{p_{l}^{j}}, \\
& \text { if the } i \text {-th student had worked on } \\
& a_{p_{l}}^{j}(i)= \begin{cases} & \text { level } p_{l}^{j}, \\
0, & \end{cases} \\
& \text { on level } p_{l}^{j} \text {, } \\
& b_{p_{l}}^{j}(i)=\left\{\begin{array}{l}
\frac{p_{l}^{j}-\left(c_{l}^{j}(i)+d_{l}^{j}(i)\right)}{p_{l}^{j}}, \\
\quad \text { if the } i \text {-th student had worked on } \\
\text { level } p_{l}^{j}, \\
0, \text { if the } i \text {-th student had not worked } \\
\text { on level } p_{l}^{j},
\end{array}\right.
\end{aligned}
$$

where

- $c_{l}^{j}(i)$ are the points for the solution of the level $p_{l}^{j}$ of the $j$-th assessment unit of the $i$-th student,

- $d_{l}^{j}(i)$ are the points for the description of the decision of the level $p_{l}^{j}$ of the $j$-th assessment unit of the $i$-th student.

Therefore, the degree of uncertainty, in this case, is equal to 1 , when the $i$-th student did not work on the level $p_{l}^{j}$ of the $j$-th assessment unit.

\subsection{Determine of the Final Mark for the $i$-th Student}

Here we introduce intuitionistic fuzzy coefficients $\langle\delta, \epsilon\rangle$, setting weights of each assessment unit that contribute to the final mark for the $i$-th student, $i=1,2, \ldots, m$. Coefficient $\delta$ is based on the number of successive assessment units, and coefficient $\epsilon$ is based on the number of preceding assessment units. An example can clarify this. Suppose, for instance, that a trainee sits for nine assessment units, divided into three levels of difficulty (easy, average, difficult). Let there be four assessment units from the first level, three assessment units from the second level, and two assessment units from the third level. Then the weight coefficients will be distributed as follows:

- from the first level: $\left\langle\frac{5}{9}, 0\right\rangle$,

- from the second level: $\left\langle\frac{2}{9}, \frac{4}{9}\right\rangle$, and

- from the third level: $\left\langle 0, \frac{7}{9}\right\rangle$.

In this way, the $(j+1)$ st intuitionistic fuzzy estimation $\left\langle\mu^{j+1}(i), \nu^{j+1}(i)\right\rangle$, is calculated on the basis of the preceding estimations $\left\langle\mu^{j}(i), \nu^{j}(i)\right\rangle$ is obtained according to the following formula (5), for $i=1,2, \ldots, m, j=1,2, \ldots, n$.

$$
\begin{aligned}
& \left\langle\mu^{j+1}(i), \nu^{j+1}(i)\right\rangle \\
& =\left\langle\frac{\mu^{j}(i) \cdot j+\delta^{j} \cdot m+\varepsilon^{j} \cdot n}{j+1}, \frac{\nu^{j}(i) \cdot j+\delta^{j} \cdot n+\varepsilon^{j} \cdot m}{j+1}\right\rangle,
\end{aligned}
$$

where $\langle m, n\rangle$ is the estimation of the current assessment unit, $m$, $n \in[0,1]$ and $m+n \leq 1$, and $\left\langle\delta^{j}, \epsilon^{j}\right\rangle$ is the weight coefficient of the $j$ th assessment unit, for $\delta^{j}, \epsilon^{j} \in[0,1], \delta^{j}+\epsilon^{j} \leq 1$.

The calculated final mark based on all assessment units for the $i$-th student has to satisfy the necessary "minimal threshold of knowledge". To check this, we introduce threshold values:

$$
\begin{aligned}
& M_{\max }, M_{\min }, N_{\max }, N_{\min } \text {. } \\
& \text { If } \\
& \mu(i)>M_{\max } \& \nu(i)<N_{\min }
\end{aligned}
$$

then the $i$-th student satisfies the "minimal threshold of knowledge" for the current e-learning course.

If

$$
\mu(i)<M_{\min } \& \nu(i)>N_{\max },
$$

then the $i$-th student does not satisfy the "minimal threshold of knowledge" for the current e-learning course and he/she has to be evaluated for all assessment units again.

In the rest of the cases the "minimal threshold of knowledge" is undefined and the $i$-th student has to be evaluated again for the assessment units for which:

$$
\mu^{j}(i) \leq M_{\max } \& \nu^{j}(i) \geq N_{\min }
$$


is valid.

\subsection{Defining the Interval Valued Intuitionistic Fuzzy Eval- uation of the Whole Student Class}

Each interval valued intuitionistic fuzzy evaluation has the form $\langle M, N\rangle$, where $M, N \subset[0,1]$ are intervals, i.e., $M=$ $[\inf M, \sup M], N=[\inf N, \sup N]$ and

$$
\sup M+\sup N \leq 1
$$

When we have some (e.g., c) student classes, we can obtain aggregated evaluation for them on the basis of the intuitionistic fuzzy evaluations of the separate students in each class, but we can obtain interval valued intuitionistic fuzzy evaluation of the class, too.

In the first case, for the $k$-th class $(k=1,2, \ldots, c)$ we obtain that the evaluation is based on formulas (1), (2) or (5) and it has the form

$$
\left\langle\mu_{k}, \nu_{k}\right\rangle=\left\langle\frac{1}{m_{k} \sum_{i=1}^{m_{k}} \mu_{k, i}}, \frac{1}{m_{k} \sum_{i=1}^{m_{k}} v_{k, i}}\right\rangle,
$$

where $m_{k}$ is the number of the students in the $k$-th class, and $\left\langle\mu_{k, i}, \nu_{k, i}\right\rangle$ is the final evaluation of the $i$-th student in the $k$-th class.

When we want to obtain interval valued intuitionistic fuzzy evaluation of the $k$-th class, we construct the numbers

$$
\begin{aligned}
& M_{k, \text { inf }}=\inf \left\{\mu_{k, i} \mid i=1,2, \ldots, m_{k}\right\}, \\
& M_{k, \text { sup }}=\sup \left\{\mu_{k, i} \mid i=1,2, \ldots, m_{k}\right\}, \\
& N_{k, \text { inf }}=\inf \left\{\nu_{k, i} \mid i=1,2, \ldots, m_{k}\right\}, \\
& N_{k, \text { sup }}=\sup \left\{\nu_{k, i} \mid i=1,2, \ldots, m_{k}\right\} .
\end{aligned}
$$

Now, we can construct the interval valued intuitionistic fuzzy evaluation

$$
\left\langle M_{k}, N_{k}\right\rangle=\left\langle\left[M_{k, \text { inf }}, M_{k, \text { sup }}\right],\left[N_{k, \text { inf }}, N_{k, \text { sup }}\right]\right\rangle .
$$

When we like to compare two classes ( $k$-th and $l$-th) on the bases of their results, we can use the following formulas (see [12]):

$$
\left\langle M_{k}, N_{k}\right\rangle \leq\left\langle M_{l}, N_{l}\right\rangle
$$

if and only if $M_{k, \text { inf }} \leq M_{l, \text { inf }}, M_{k, \text { sup }} \leq M_{l, \text { sup }}, N_{k, \text { inf }} \geq N_{l, \text { inf }}, N_{k, \text { sup }} \geq N_{l, \text { sup }}$. e-learning evaluation with intuitionistic fuzzy estimations,"
In [12], other formulas for evaluation are discussed, too.

\section{Conclusion}

In the current paper we have presented the procedure that gives the possibility for the algorithmization of the method of forming the student's evaluations by applying intuitionistic fuzzy estimations. The suggested evaluation methodology and procedures are intended to make the student's evaluations as objective as possible.

In practice, subjective estimation cannot be entirely avoided but it should be made as objective as possible. This can be achieved, to some extent, by approaches which employ quanti-

In addition, the discussed procedure can be extended for arbitrary facts having intuitionistic fuzzy or interval valued intuitionistic fuzzy evaluations, using the same approach. The procedure can be described by a generalized net and this possibility will be in accord with the idea from [4] that the apparatus of the intuitionistic fuzziness can be used successfully for evaluation of data mining processes.

\section{Conflict of Interest}

No potential conflict of interest relevant to this article was reported.

\section{Acknowledgement}

This work was supported by the National Science Fund of Bulgaria (Grant No. DN-I-02-10, New instruments for knowledge discovery from data, and their modelling). The first author's work in this paper was conducted during the sabbatical year of Kwangwoon University in 2018.

\section{References}

[1] Kensington-Miller, J. Novak, and T. Evans, "Just do it: flipped lecture, determinants and debate," International Journal of Mathematical Education in Science and Technology, vol. 47, no. 6, pp. 853-862, 2016. https: //doi.org/10.1080/0020739X.2015.1129075

[2] P. Melo-Pinto, T. Kim, K. Atanassov, E. Sotirova, A Shannon, and M. Krawczak, "Generalized net model of tative methods to utilize the instruments of subjective statistics. 
in Proceedings of the 1st International Workshop on Intuitionistic Fuzzy Set and Generalized Nets (IWIFSGN), Warszawa, Poland, 2005, pp. 241-249.

[3] A. Shannon, K. Atanassov, D. Langova-Orozova, M. Krawczak, E. Sotirova, P. Melo-Pinto, I. Petrounias, and T. Kim, Generalized Net Modelling of University Processes. Sydney: KvB Institute of Technology, 2005.

[4] A. Shannon, K. Atanassov, D. Langova-Orozova, M. Krawczak, E. Sotirova, P. Melo-Pinto, I. Petrounias, and T. Kim, Generalized Nets and Information Flow Within a University. Warszawa: Warsaw School of Information Technology, 2007.

[5] A. Shannon, E. Sotirova, I. Petrounias, K. Atanassov, M. Krawczak, P. Melo-Pinto, and T. Kim, "Intuitionistic fuzzy estimations of lecturers' evaluation of student work," in Proceedings of the 1st International Workshop on Intuitionistic Fuzzy Sets, Generalized Nets \& Knowledge Engineering, London, 2006, pp. 44-47.

[6] A. Shannon, E. Sotirova, I. Petrounias, K. Atanassov, M. Krawczak, P. Melo-Pinto, and T. Kim, "Generalized net model of lecturers' evaluation of student work with intuitionistic fuzzy estimations," in Proceedings of the 2nd International Workshop on Intuitionistic Fuzzy Sets, Banska Bystrica, Slovakia, 2006, pp. 22-28.

[7] A. Shannon, E. Sotirova, K. Atanassov, M. Krawczak, P. Melo-Pinto, and T. Kim, "Generalized net model for the reliability and standardization of assessments of student problem solving with intuitionistic fuzzy estimations," in Proceedings of the 8th International Workshop on Intuitionistic Fuzzy Sets and Generalized Nets (IWIFSGN), 2009, Warsaw, Poland, pp. 249-256.

[8] A. Shannon, D. Dimitrakiev, E. Sotirova, M. Krawczak, and T. Kim, "Towards a model of the digital university: generalized net model of a lecturer's evaluation with intuitionistic fuzzy estimations," Cybernetics and Information Technologies, vol. 9, no. 2, pp. 69-78, 2009.

[9] A. Shannon, E. Sotirova, M. Hristova, and T. Kim, "Generalized net model of a student's course evaluation with intuitionistic fuzzy estimations in a digital university," Proceedings of the Jangjeon Mathematical Society, vol. 13, no. 1, pp. 31-38, 2010.
[10] E. Sotirova, K, Atanassov, A. Shannon, M. Krawczak, Melo-Pinto, B. Riecan, and T. Kim, "Intuitionistic fuzzy evaluations for analysis of a student's knowledge of mathematics in university e-learning courses," in Proceedings of IEEE 8th International Conference on Intelligent Systems, 2016, pp. 535-537. https://doi.org/10.1109/IS.2016. 7737474

[11] K. Atanassov, Generalized Nets. Singapore: World Scientific Publishing, 1991.

[12] K. Atanassov, On Intuitionistic Fuzzy Sets Theory. Heidelberg: Springer, 2012.

[13] K. Atanassov, "Intuitionistic fuzzy logics as tools for evaluation of data mining processes," Knowledge-Based Systems, vol. 80, pp. 122-130, 2015. https://doi.org/10.1016/j. knosys.2015.01.015

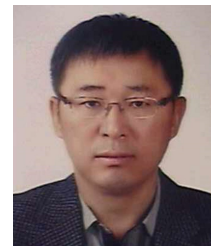

Taekyun Kim is a full professor at Department of Mathematics, Kwangwoon University, Seoul, Korea. He is Chair Professor at Department of Mathematics, Tianjin Polytechnic University, Tianjin, China. He received Ph.D. at Kyushu University, Fukuoka, Japan(1989-1994). His research interests are number theory, mathematical physics, mathematical modeling, fuzzy sets, intelligent system.

E-mail: tkkim@kw.ac.kr

Evdokia Sotirova is a professor in Intelligent Systems Laboratory, University "Prof. Dr. Assen Zlatarov", Burgas, Bulgaria. Her principal research interests are generalized nets modelling data mining.

E-mail: esotirova@btu.bg

Anthony Shanno is a professor in Warrane College, The University of New South Wales, Kensington, Australia. His principal research interests are number theory, mathematical modeling, fuzzy sets.

E-mail: t.shannon@warrane.unsw.edu.au

Vassia Atanassova is a professor in Department of Bioinformatics and Mathematical Modelling, Institute of Biophysics and Biomedical. Ph.D. degrees in Informatics and Computer Sciences, Institute of Information and Communication Technologies BAS (2013). His principal research interests are bioinformatics and mathematical modelling. 
E-mail: vassia.atanassova@gmail.com

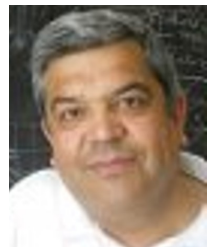

Krassimir Atanassov is a professor in Engineering, Bulgarian Academy of Sciences, Sofia, Bulgaria. His principal research interests are mathematical modelling artificial intelligence intuitionistic fuzzy sets, generalized nets, and number theory.

E-mail: krat@bas.bg
Lee-Chae Jang is a professor in Graduate School of Education, Konkuk University, Korea. He received his B.S., M.S., and Ph.D. degrees in the department of mathematics from Kyungpook National University, Korea. He was a visiting professor at Cincinnati University, USA. His principal research interests are Fuzzy theory, Analysis, and p-adic analysis.

E-mail: Lcjang@konkuk.ac.kr 